ARTICLE

Received 26 Aug 2013 | Accepted 18 Feb 2014 | Published 17 Mar 2014

DOI: $10.1038 /$ ncomms 4473

\title{
Bulk mixed ion electron conduction in amorphous gallium oxide causes memristive behaviour
}

\author{
Yoshitaka Aoki ${ }^{1 \star \star}$, Carsten Wiemann², Vitaliy Feyer ${ }^{2}$, Hong-Seok Kim³ ${ }^{3}$, Claus Michael Schneider ${ }^{2,4}$, \\ Han III-Yoo ${ }^{3} \&$ Manfred Martin ${ }^{1,3, *}$
}

In thin films of mixed ionic electronic conductors sandwiched by two ion-blocking electrodes, the homogeneous migration of ions and their polarization will modify the electronic carrier distribution across the conductor, thereby enabling homogeneous resistive switching. Here we report non-filamentary memristive switching based on the bulk oxide ion conductivity of amorphous $\mathrm{GaO}_{x}(x \sim 1.1)$ thin films. We directly observe reversible enrichment and depletion of oxygen ions at the blocking electrodes responding to the bias polarity by using photoemission and transmission electron microscopies, thus proving that oxygen ion mobility at room temperature causes memristive behaviour. The shape of the hysteresis I-V curves is tunable by the bias history, ranging from narrow counter figure-eight loops to wide hysteresis, triangle loops as found in the mathematically derived memristor model. This dynamical behaviour can be attributed to the coupled ion drift and diffusion motion and the oxygen concentration profile acting as a state function of the memristor.

\footnotetext{
${ }^{1}$ Institute of Physical Chemistry, RWTH Aachen University and JARA-FIT, 52056 Aachen, Germany. ${ }^{2}$ Peter Grünberg Institute (PGI-6) and JARA-FIT, Research Centre Jülich, 52425 Jülich, Germany. ${ }^{3}$ Department of Materials Science and Engineering, Seoul National University, Seoul 151-744, Korea.

${ }^{4}$ Fakultät für Physik and Center for Nanointegration Duisburg-Essen (CENIDE), Universität Duisburg-Essen, D-47048 Duisburg, Germany. ${ }^{\star}$ These authors contributed equally to this work. Correspondence and requests for materials should be addressed to M.M. (email: martin@rwth-aachen.de).
} 
A memristor is a resistive switching device that varies its internal resistance state according to the history of the applied voltage and current ${ }^{1-3}$. Following the definition by Chua et $a .^{1,2}$, it can be described by a set of equations including the current equation, $V=R(w, I) I$, and a rate equation, $\mathrm{d} w / \mathrm{d} t=f(w, V)$. Here $V, I$ and $R$ are the voltage, current and resistance, respectively. $w$ is an internal state variable, described by a time-varying function $f$, and it is crucial to the switching dynamics of the memristor. The time-dependent switching suggests that a memristor could be a physical system that enables to emulate the synaptic memory function more directly than the complementary metal-oxide semiconductor system ${ }^{4-7}$. Recently, memristive resistive switching of many transition metal-oxide-based semiconductor films has been extensively studied $^{4,8-10}$. The formation ${ }^{9-14}$ or modulation ${ }^{15-18}$ of conductive channels, tens or hundreds of nanometres in diameter, driven by the drift/diffusion of oxygen vacancies are believed to play a key role in the mechanism. In case of the wellestablished $\mathrm{Pt} / \mathrm{TiO}_{2} / \mathrm{Pt}$ memristor devices, the state variable $w$ is the tunnel barrier width between a top electrode (TE) and the preformed conductive filament, which is modified by the ionic drift within a few nanometre-thick space charge layer. The shift of ions in such a confined space must immediately equilibrate and the relaxation between high-resistive state (HRS) and lowresistive state (LRS) terminates within a few microseconds ${ }^{16,19,20}$.

In principle, mixed ionic electronic conductors (MIEC) sandwiched by two blocking electrodes may show non-filamentary resistive switching, if the ions are uniformly conductive throughout the film bulk, even at room temperature, since the homogeneous migration of ions will modify the electronic carrier distribution across the conductor, and thus its bulk resistivity $3,21-23$. The bulk diffusion of dopants may require longer decay times and larger changes of bulk states until equilibrium than the ionic motion in a confined space. The suggested time dependency and number of resistance states in non-filamentary resistive switches are quite different from those in filamentary devices $5,7,24,25$. However, there are only very few well-known bulk memristive systems ${ }^{25-28}$, since the bulk ionic conductivity of MIECs is usually very low near room temperature. Furthermore, the direct evidence for the bulk state function of the switching system is still missing. Hence, it is a great challenge for material scientists to establish a nonfilamentary-type MIEC memristor based on the efficient, bulk ion conduction at low temperatures.

Herein, we demonstrate the homogeneous resistive switching in mixed oxide ion electron-conducting, amorphous gallium oxide thin films, $a-\mathrm{GaO}_{x}$, with the main state function being the timedependent oxygen concentration profile across the film. The film possesses nonlinear, dynamical switching behaviour as is observed in the mathematically derived memristor model ${ }^{2,3,21}$, in which the $I-V$ responses can be varied from a continuous counter figure-eight loop at high frequencies to a quasi-abrupt, triangle-like switching loop at lower frequencies, as depending on the bias history. This is the first report of the efficient oxide ion conductivity of an amorphous main-group oxide thin film at room temperature, perhaps opening up a new route to obtain dynamical, homogeneous switching memristor systems. The main objective of this study is to demonstrate the basic working principle of the homogeneous $\mathrm{GaO}_{x}$ switching device, while technical characterization of the device is beyond the scope of this manuscript.

\section{Results}

Resistive switching behaviour. Previously, we reported on the synthesis of highly nonstoichiometric, amorphous gallium oxide, $a-\mathrm{GaO}_{x}(x \sim 1.1)$, by a pulsed laser deposition (PLD) ${ }^{29}$. This oxide is-due to its high gallium excess-a heavily self-doped, $n$-type semiconductor ${ }^{29}$. Recently, several authors reported that $a-\mathrm{GaO}_{x}$ thin films with Pt as TE and ITO or TiN as bottom electrode (BE) reveal a counter figure-eight hysteretic loop in their $I-V$ characteristics $^{30,31}$. They proposed a filamentary switching mechanism, similar to that introduced for $\mathrm{TiO}_{x}$, to explain $\mathrm{GaO}_{x}$ switching ${ }^{30-32}$. However, there are qualitative differences in the memristive behaviour between $\mathrm{GaO}_{x}$ and $\mathrm{TiO}_{x}$, as will be shown below. Thus, we propose a non-filamentary resistive switching mechanism for $\mathrm{GaO}_{x}$ that is consistent with both the structure of the films and the observed switching dynamics.

We first conducted a systematic study of the $I-V$ characteristics by changing the film thicknesses, the voltage-sweep rates and the temperatures. For this purpose, we fabricated a film device of Pt$\mathrm{TE}(100 \mathrm{~nm}) / \mathrm{GaO}_{x} / \mathrm{ITO}-\mathrm{BE}(40 \mathrm{~nm})$ by depositing amorphous $\mathrm{GaO}_{x}(x \sim 1.1)$ films by PLD at the same conditions as described in our previous report ${ }^{29}$. The chemical composition was checked by using scanning transmission electron microscope equipped with energy dispersive X-ray spectrometer (STEM-EDX). A typical $I-V$ curve for a $90-\mathrm{nm}$-thick film is shown in Fig. 1a. The pristine $\mathrm{GaO}_{x}$ film clearly reveals a pinched hysteretic loop with counter figure-eight polarity as sweeping the voltage at the TE in the way of $0 \rightarrow+2 \rightarrow 0 \rightarrow-2 \rightarrow 0 \mathrm{~V}$, indicating memristive behaviour of the film. A positive voltage (about $+1.7 \mathrm{~V})$ gradually increases the resistance so as to SET the film from the LRS to the HRS, and with negative bias $(-2 \mathrm{~V})$ the film is RESET to the LRS. This bipolar behaviour is stationary after the 1st sweep cycle, revealing that the pristine, homogeneous state (corresponding to the as-prepared film) is not recovered after the 1st cycle. We emphasize that the $\mathrm{GaO}_{x}$ device does not require any electrical-forming process in contrast to $\mathrm{TiO}_{x}$-based devices where forming of filaments is a prerequisite ${ }^{9,33}$. This is a great advantage because the device reliability is independent on the reproducibility of the microstructural filament formation.

The homogeneous resistive switching mechanism is clearly demonstrated by two experimental findings shown in Fig. 1b,d. First, the current levels of our $a-\mathrm{GaO}_{x}$ device linearly scale with the TE area (Fig. 1b; Supplementary Fig. 1). Second, we observe the wide variety of hysteretic shapes in the $I-V$ curves, depending on the voltage-sweep rate $v_{\text {sw }}$ and the film thickness $L$ (Fig. 1d) The films reveal counter figure-eight loops, triangular loops, quasi-abrupt switching like loops and even non-hysteretic $I-V$ curves. The hysteretic area in the $I-V$ curves increases with decreasing film thickness and decreases with increasing voltagesweep rate. Both observations are supportive for the homogeneous resistive switching mechanism triggered by bulk ion conduction and rule out a filamentary mechanism: First, as shown in Supplementary Fig. 1 the current density at the TE is independent on the TE area. Second, when the film thickness and/or the voltage-sweep rate increases no appreciable polarization of the ions can build up during one sweep cycle, and thus the hysteretic area decreases. Once the hysteretic area becomes very small, for example, in the 90 -nm-thick film at high sweep rates, a nonlinear $I-V$ curve is observed. This is probably due to the tunnelling nature of the interface between the high work function Pt electrode (5.6 eV (ref. 34)) and the $n$-type gallium oxide ${ }^{29}$. Higher bias amplitudes also result in the transformation of the hysteretic loop in the anodic region from an ellipsoidal loop to a triangular one (Fig. 1c). Finally, we emphasize that all changes of the $I-V$ curves shown in Fig. 1c,d are completely reversible (see Supplementary Fig. 2), showing that the films do not experience irreversible changes during switching. The complete series of $I-V$ curves as function of film thickness, voltage-sweep rate and temperature as well is given in Supplementary Fig. 3.

Figure 2 shows the current transient when a constant $-1.7 \mathrm{~V}$ bias is suddenly applied on the pristine film at $t=0$. The negative 
a

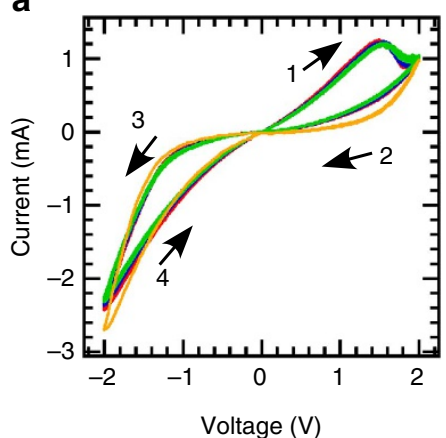

b



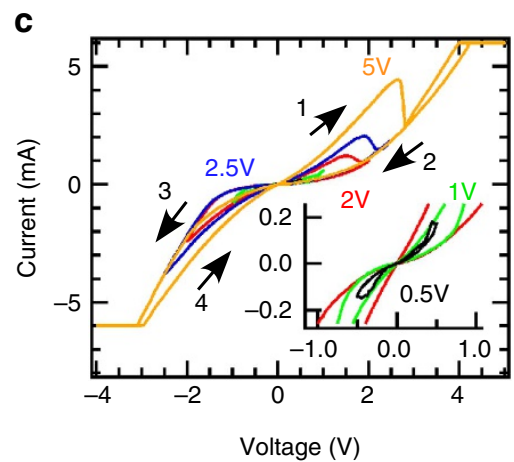

d

\begin{tabular}{llllll}
\multicolumn{7}{c}{$v_{\text {sw }}\left(\mathrm{V} \mathrm{s}^{-1}\right)$} \\
\hline 0.005 & 0.05 & 0.5 & 5 & 50 & 500
\end{tabular}
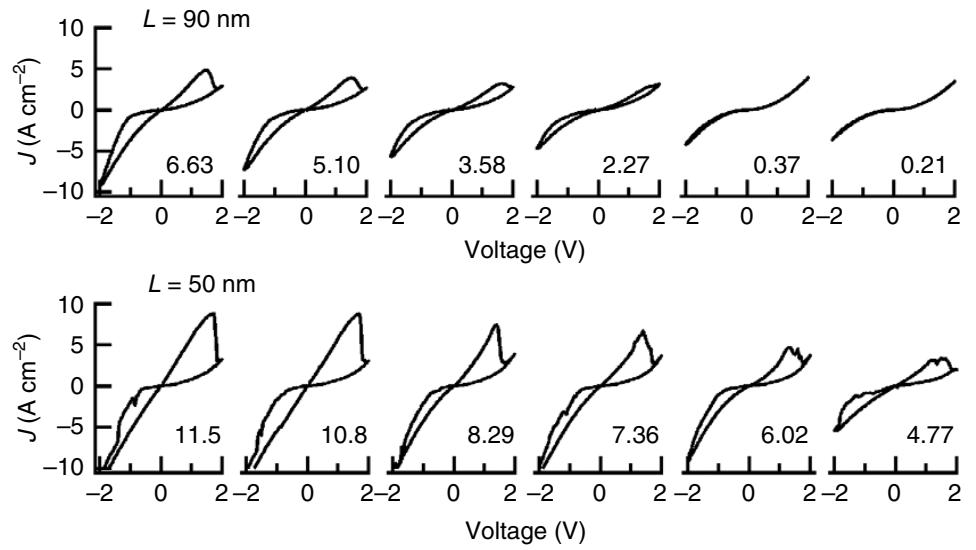

Figure 1 | Bipolar resistive switching of Pt(TE)/a-GaO $\mathbf{x} / \mathbf{I T O}$ (BE) devices. (a) I-V characteristics of a 90-nm a-GaO film during a 30-times voltagesweep cycle of $0 \rightarrow+2 \rightarrow-2 \rightarrow 0 \mathrm{~V}$ at a sweep rate $v_{\mathrm{sw}}=0.05 \mathrm{~V} \mathrm{~s}^{-1}$ and a temperature of $25^{\circ} \mathrm{C}$. The curves measured with $\mathrm{TE}$ diameter $=200 \mu \mathrm{m}$ and $\mathrm{BE}$ area $=1 \times 1 \mathrm{~cm}^{2}$ for the 1st sweeping cycle (yellow), 2-10th cycles (red), 11-20th cycles (blue) and 20-30th cycles (green). The numbered arrows indicate the direction of the switching cycles (counter figure-eight loops). (b) TE area scaling of the current of LRS at $-1.7 \mathrm{~V}$ (white triangles) and HRS at $+1.7 \mathrm{~V}$ (black triangles). The solid lines for both plots show linear scaling with a slope of 1. (c) $1-\mathrm{V}$ characteristics of $90 \mathrm{~nm}$ films measured at $v_{\mathrm{sw}}$ of $0.05 \mathrm{Vs}^{-1}$ with various bias amplitudes. (d) Variable hysteretic $1-\mathrm{V}$ loops of $a-\mathrm{GaO}_{1.1}$ thin films with thickness of 50 and $90 \mathrm{~nm}$, measured at $25^{\circ} \mathrm{C}$ with various voltage-sweep rates $v_{\mathrm{sw}}$ in the range of $0.005-500 \mathrm{Vs}^{-1}$. The numbers in each graph denote the hysteretic area $\left(\mathrm{VAcm}^{-2}\right)$ of the loop. $a-\mathrm{GaO}_{x}$ thin films can reveal non-hysteresis curves, counter figure-eight loops, triangular loops and quasi-abrupt switching-like loops as depending on $v_{\text {sw. }}$

current exponentially increases with increasing the bias duration, indicating that the resistance state of the pristine film decays exponentially to the LRS by cathodic polarization. The relaxation of the 90 -nm-thick film apparently becomes faster with elevating temperature. The relaxation curves at each temperature can be described well by a stretched exponential relaxation function, which is represented by $\exp \left\{-(\tau / t)^{\beta}\right\}$. This result discloses that the resistive switching of $a-\mathrm{GaO}_{x}$ is triggered by ion conduction and redistribution within its glassy matrix. The stretched exponential relaxation originates from the wide distribution of activation energies for ion conduction and the associated wide range of relaxation times among different relaxation processes in a disordered system ${ }^{35-38}$. Ion conductivity relaxation of many oxide glasses obeys this function because the structural inhomogeneity provides widely distributed activation energies for site-to-site hopping ${ }^{36-38}$. Here the Kohlrausch stretching exponent $\beta$ ranging from 0 to 1 gives the correlation between the activation energy of ion conductivity $E_{\mathrm{a}}{ }^{*}$ and average microscopic energy barrier $E_{\text {a }}$ impeding the ion movement between sites as defined by $E_{\mathrm{a}}=\beta E_{\mathrm{a}}{ }^{*}$ (refs 36-38), and the relaxation time $\tau$ is proportional to $\varepsilon_{0} \varepsilon_{\mathrm{s}} \sigma_{\mathrm{dc}}{ }^{1}$ with $\varepsilon_{0}$ as permittivity of vacuum, $\varepsilon_{\mathrm{s}}$ dielectric constant of the glass and $\sigma_{\mathrm{dc}}$ its ion conductivity ${ }^{36}$. Empirically, it has been known that higher conductivity gives lower $\beta$ and $\tau$ (refs 36,37). For cation conducting glasses with $\sigma_{\mathrm{dc}}$ of $\sim 10^{-4} \mathrm{~S} \mathrm{~cm}^{-1}, \beta$ and $\tau$ are about 0.6 (ref. 38) and about $10 \mathrm{~ns}$ (ref. 37), respectively. The fitting for the 90 -nm-thick film gives $\beta$ of about 0.9 and $\tau$ of $>0.01 \mathrm{~s}$ at each temperature, revealing that the oxide ion conduction is rather low at the measured temperature range, as necessary for long retention times. The Arrhenius plot of $\tau$ yields an activation energy of $E_{\mathrm{a}}{ }^{*}=0.5 \mathrm{eV}$ for the ion conductivity (Supplementary Fig. 4). As the ion conductivity increases with increasing temperature, the hysteretic loop of the $I-V$ characteristics is clearly enlarged (inset of Fig. 2).

Valence change by homogeneous oxide ion migration. As a consequence of the electrochemical polarization of the $\mathrm{GaO}_{\mathrm{x}}$ films the Ga valence must change as a function of the applied bias. In order to identify the Ga valence states in the HRS and the LRS micrometre probe X-ray photoelectron spectroscopy ( $\mu$-XPS) was performed on the bare surface of polarized films after removing the TE (Fig. 3a). Ga 3D photoemission electron microscopy (PEEM) images taken at a binding energy of $20.6 \mathrm{eV}$ (Fig. 3b) clearly differentiate between the HRS film within the TE mark area (bright) and the pristine surface area (grey), thus indicating 


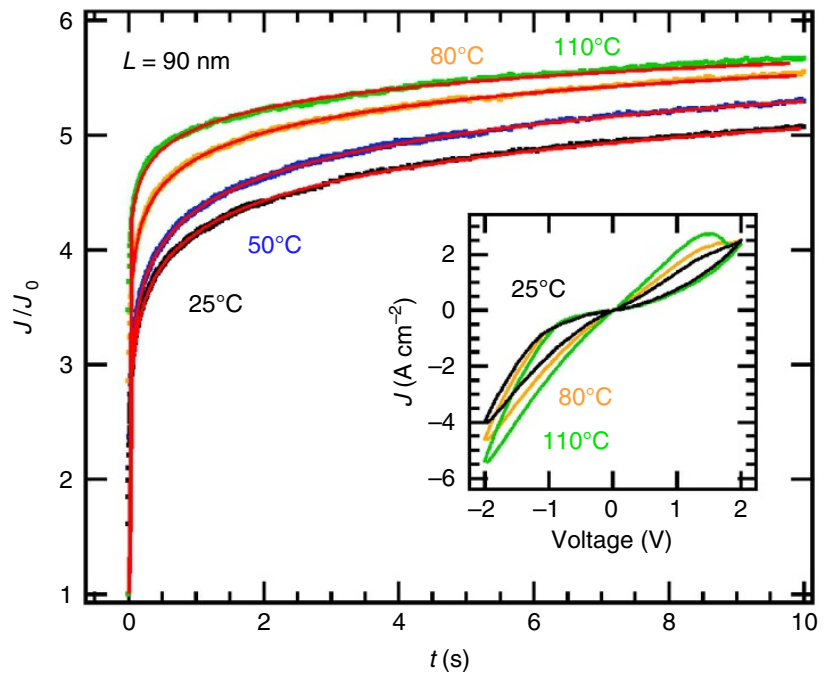

Figure 2 | Current transients of $a-\mathrm{GaO}_{x}$ films during resistive switching from HRS to LRS. Current transients of 90 -nm-thick pristine films after applying a bias of $-1.7 \mathrm{~V}$ to the TE at $t=0$. The coloured dots are the measured values, and the red lines are the fits by a stretched exponential function, $J / J_{0}=A \exp \left\{-(t / \tau)^{\beta}\right\}$, with fitting parameters of pre-exponential factor $A$, relaxation time $\tau(\mathrm{s})$ and Kohlrausch stretching exponent $\beta$ (for details see main text). Inset: temperature dependence of the $I-V$ characteristics of 90-nm-thick films, measured at $v_{\mathrm{sw}}=0.5 \mathrm{~V}-\mathrm{s}^{-1}$. Best fitting for the $90 \mathrm{~nm}$ films at $25,50,80$ and $110^{\circ} \mathrm{C}$ was obtained with a set of parameters $(A, \tau, \beta)=(10.1,0.24,0.88),(9.4,0.20,0.88),(9.8,0.057$, $0.90)$ and $(10.6,0.011,0.93)$, respectively.

differences in the Ga valence states between the polarized and the unpolarized regions. The corresponding spectra (red and blue lines in the inset of Fig. 3b) more clearly demonstrate that the Ga valence state changes by polarization. Figure $3 c$ shows the $\mu$-XPS spectra collected on the pristine and polarized film surfaces, respectively. The pristine film exhibits three different $\mathrm{Ga}$ valence states; a strong peak at $20.7 \mathrm{eV}\left(\mathrm{P}_{1}\right)$ is assigned to the oxide $\left(\mathrm{Ga}^{3+}\right)^{39}$ state, a peak at around $19.6 \mathrm{eV}\left(\mathrm{P}_{2}\right)$ to the suboxide $\left(\mathrm{Ga}^{+}\right)^{39,40}$ state and a pair of small peaks at $18.7\left(\mathrm{P}_{3}\right)$ and $18.2 \mathrm{eV}$ $\left(\mathrm{P}_{4}\right)$ can be assigned to the metal $\left(\mathrm{Ga}^{0}\right)$ state (by comparing with a GaCo alloy reference sample, Supplementary Fig. 5). The average valence state of $\mathrm{Ga}$ in the pristine film is calculated to be +2.3 (Table 1), in good agreement with the value +2.2 that is calculated from the composition of the film, $\mathrm{GaO}_{1.1}$, obtained by STEM-EDX.

It appears that the Ga valence state within the whole TE mark area is homogeneously switched by anodic or cathodic polarization. The valence state of $\mathrm{Ga}$ is variable between $\mathrm{Ga}^{3+}$ and $\mathrm{Ga}^{+}$ and the metallic state does not evolve again after the 1st sweep cycle. Therefore, the different behaviour in the 1st voltage-sweep cycle (see Fig. 1a) is due to the oxidation of $\mathrm{Ga}^{0}$ to $\mathrm{Ga}^{+}$and $\mathrm{Ga}^{3+}$. In the 2 nd sweep cycle, when sweeping the voltage to $+2 \mathrm{~V}$ (SET the film to HRS) $>80 \%$ of Ga exists in the highest oxidation state $\left(\mathrm{Ga}^{3+}\right)$ as calculated from the $\mathrm{P}_{1} / \mathrm{P}_{2}$ peak area ratio, and so the average oxidation state of $\mathrm{Ga}$ increases from +2.3 in the pristine film to +2.7 (Table 1). RESET to the LRS by negative biasing $(-2 \mathrm{~V})$ reduces $\mathrm{GaO}_{x}$ (with about $50 \%$ of $\mathrm{Ga}$ in the suboxide state $\mathrm{Ga}^{+}$) so as to complete the $\mathrm{Ga}$ redox cycle. These results strongly indicate that positive or negative bias on the TE homogeneously oxidizes or reduces the $\mathrm{GaO}_{x}$ underlayer, respectively, by migration of oxygen ions (or oxygen vacancies) towards or away from the TE. This must cause the modification of the donor density in the underlayer since oxygen vacancies act as donors in $\mathrm{GaO}_{x}$ (ref. 29). In fact, the near-Fermi edge spectra in Fig. 3d show that the density of states near the VB edge is also variable throughout the redox cycle of the film; that of a pristine film is decreased by oxidation at $+2 \mathrm{~V}$, but is increased again to the similar level as that of the pristine film by reduction at $-2 \mathrm{~V}$. Such a Ga redox cycle is clearly reproducible in the 3rd sweep cycle. Furthermore, the molar ratio of $\mathrm{Ga}^{+} / \mathrm{Ga}^{3+}$ continuously increases in proportion to the negative bias amplitude as $0.3 / 0.7$ at $-1.3 \mathrm{~V}$ and $0.5 / 0.5$ at $-2 \mathrm{~V}$ (Fig. $3 \mathrm{~d}$ ), indicating that the oxidation state of $\mathrm{GaO}_{x}$ is continuously variable. Finally, we emphasize that it would be impossible to detect the observed $\mathrm{Ga}$ valency changes in the polarized regions for filamentary switching, as the cross-section area of one or a few filaments would be by far too small to cause changes in the XPS signals originating from the large cross-section area of the exciting X-ray beam (Supplementary Fig. 6).

Figure 4 shows the STEM-EDX images of a cross-section of a 90-nm film that was polarized to a cathodic or anodic direction. Even after the polarization to $+4 \mathrm{~V}$ or $-4 \mathrm{~V}$, no heterogeneous microstructure consisting of crystalline domains or metallic filaments could be found in $>10$ examined specimens. EDX elemental mapping images clearly elucidate that oxygen is segregated within the film near the TE/film boundary by polarization to $+4 \mathrm{~V}$, but the segregation is diminished after polarization to $-4 \mathrm{~V}$ (Fig. $4 \mathrm{a}, \mathrm{b}$ ). Furthermore, the interfacial layer is uniformly enriched and depleted by oxygen according to the bias direction, revealing the non-filamentary switching behaviour. Figure $4 \mathrm{c}$ shows the oxygen and gallium line profiles across the films. The films polarized at $+4 \mathrm{~V}$ are comprised of three O-concentration regions: an $\mathrm{O}$-enriched region near the TE/film boundary within a thin, about 10-nm-wide layer (red-filled area in Fig. 4c), a region with low O-concentration at intermediate depths ranging from 10 to $30 \mathrm{~nm}$ and a middle $\mathrm{O}$-concentration region in the depth range of 40 to $90 \mathrm{~nm}$. In contrast, the $\mathrm{Ga}$ profile is kept flat throughout the film. When subsequently negative bias is applied to the TE, the O-enrichment near the TE/film boundary is decreased (compare red- and blue-filled areas), and the concentration in the intermediate depth region is slightly increased. This observation clearly shows that the resistive switching of $\mathrm{GaO}_{x}$ is caused by mobile oxygen ions and the modulation of the oxygen concentration profile, as a result of electrochemical polarization due to the applied voltage.

Numerical simulation by mixed oxide ion electron conduction. The above experimental findings unambiguously demonstrate that in our $\mathrm{GaO}_{x}$ films a whole oxide layer below the TE is homogeneously oxidized/reduced. These features do not adapt to the filamentary switching driven by locally occurring ion migration. In addition, the STEM-EDX measurements disclose that the bulk oxide ion conductivity is essential for the resistive switching of $\mathrm{GaO}_{x}$. Here, we provide a physical model for the homogeneous switching based on mixed oxide ion electron conduction under an applied electrochemical potential gradient. This model assumes that the resistive switching is due to the modulation of the carrier concentration profiles in the amorphous $n$-type semiconductor by redistribution of the oxygen vacancy donor. As our model calculations can reproduce the experimentally observed $I-V$ curves very well, we believe that the interfacial effect, that is, the Schottky barrier at the $\mathrm{Pt} / \mathrm{GaO}_{x}$ interface is of minor importance in $\mathrm{GaO}_{x}$, and thus it is omitted in our model for simplification. In our simulations the relations of the dimensions of the TE and the $\mathrm{BE}$ are similar to those of the test samples (the former with a diameter of $200 \mu \mathrm{m}$ and the latter with an area of $1 \times 1 \mathrm{~cm}^{2}$ ), which enables calculating the effect of in-plane diffusion according to the concentration gradients. The detailed explanation of the 
a
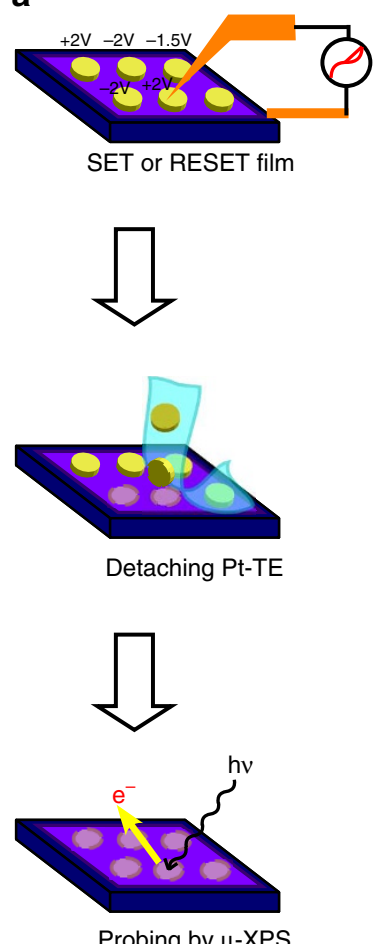

b

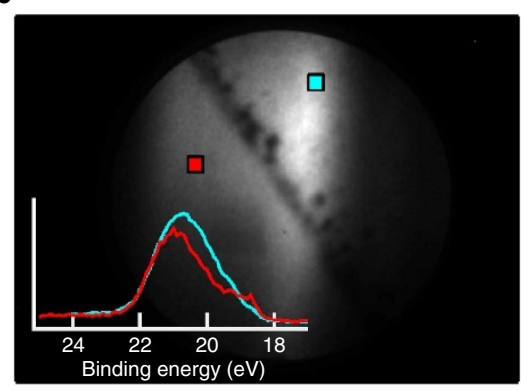

d

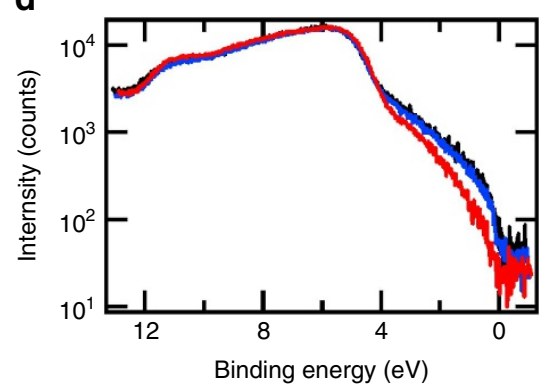

C

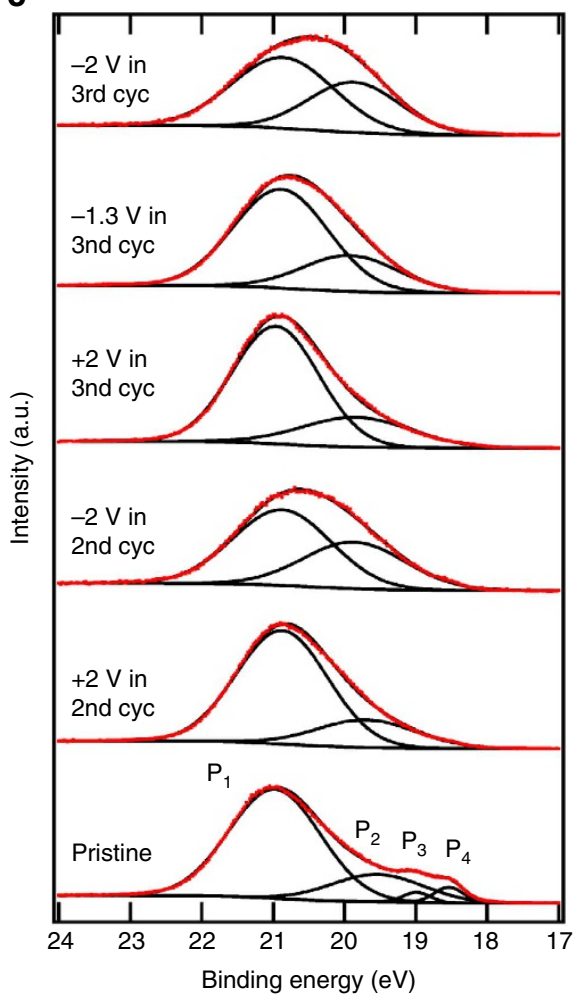

Figure 3 | Homogeneous $\mathbf{G a}$ valence state change through resistive switching of $\boldsymbol{a}-\mathbf{G a O}_{\mathbf{x}}$ device. (a) Procedure to prepare the bare $a-\mathrm{GaO}_{\mathbf{x}}$ surface after polarization to the HRS or LRS. After polarization, UV set epoxy resin was pasted on the TE, and the TE was removed by scratching off the epoxy resin. (b) Photoemission electron microscopy (PEEM) image in the Ga 3d binding energy region, measured at the border of the pristine surface (bright grey region with red square) and the bare surface of HR film under the $+2 \mathrm{~V}$-biased TE mark (diameter $=200 \mu$ m) (dark grey region with blue square). The black line along the border is due to segregation of contaminants such as Pt and resin residues. Inset: XPS spectra were reconstructed from the PEEM images at the red point within the pristine surface area (red line) and at the blue point within the polarized surface area (blue line). (c) $\mu$-XPS in the Ga $3 d$ binding energy region measured on the pristine surface and at marks of the TE polarized at different voltages during the 2 nd and $3 r d$ sweep cycle. In the polarized films, the measured spectrum (red dots) can be described well by two peaks (black line) corresponding to $\mathrm{Ga}^{3+}\left(\mathrm{P}_{1}\right)$ and $\mathrm{Ga}{ }^{+}\left(\mathrm{P}_{2}\right)$, that is, $\mathrm{Ga}$ is highly reduced. A couple of peaks corresponding to metallic $\mathrm{Ga}^{0}\left(\mathrm{P}_{3}\right.$ and $\left.\mathrm{P}_{4}\right)$ are involved only in the unpolarized, pristine film. Molar ratios of $\mathrm{Ga}^{3+} / \mathrm{Ga}^{+}$ are $0.16 / 0.84$ at $2 \mathrm{~V}$ and $0.52 / 0.48$ at $-2 \mathrm{~V}$ in the $2 \mathrm{nd}$ sweep cycle and $0.19 / 0.81$ at $2 \mathrm{~V}, 0.28 / 0.71$ at $-1.3 \mathrm{~V}$ and $0.50 / 0.50$ at $-2 \mathrm{~V}$ in the $3 \mathrm{rd}$ sweep cycle, as calculated by peak fitting analysis. (d) Near-Fermi edge spectra of a- $\mathrm{GaO}_{x}$ film: pristine (black), HRS (at $+2 \mathrm{~V}$ ) (red) and LRS (at $-2 \mathrm{~V}$ ) (blue). The density of states near the top of the valence band is variable by negative or positive biasing.

Table 1 | Summary of $\mathrm{Ga} 3 \mathrm{~d} \mu-\mathrm{XPS}$ on polarized $a-\mathrm{GaO}_{x}$ films.

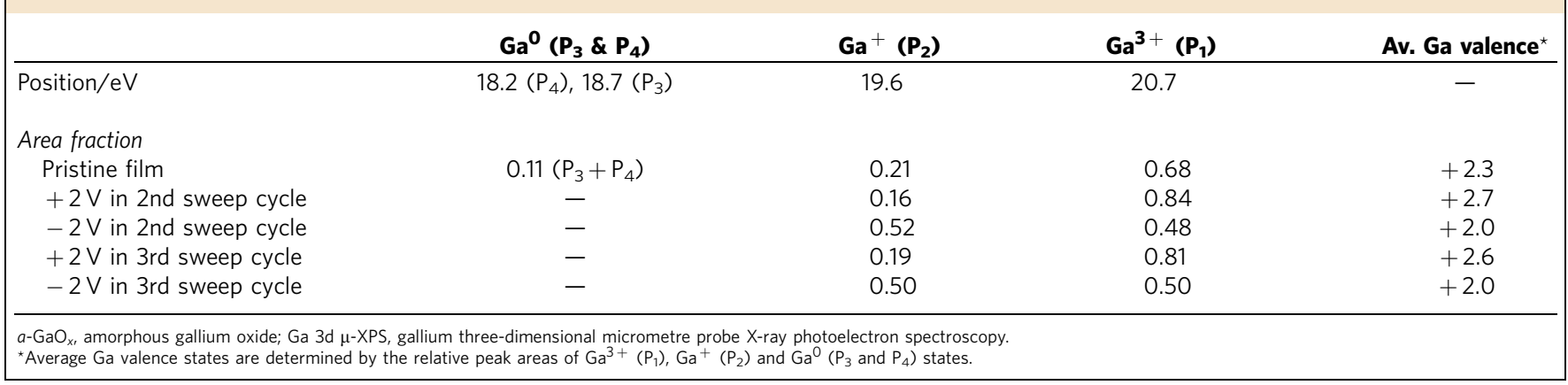

model and the simulation procedure are given in the Methods section.

The simulation semiquantitatively replicates the experimentally observed $I-V$ characteristics, exhibiting an asymmetric pinched loop in a bipolar manner (counter figure-eight loop) by switching from LRS to HRS in the anodic region and from HRS to LRS in the cathodic region. Typical simulation results are shown for the 2nd sweep cycle of a 90-nm-thick film in Fig. 5. Best agreement between experiment and simulation was obtained with a vacancy mobility $\mu_{\mathrm{V}}=7 \times 10^{-19} \mathrm{~m}^{2} \mathrm{~V}^{-1} \mathrm{~s}^{-1}$. All other parameters were fixed as specified in the Methods section. In addition to the $I-V$ curve, the calculated oxygen vacancy profiles across the film and the corresponding electronic conductivities are shown at different stages of electrochemical polarization. The electronic conductivity 
a
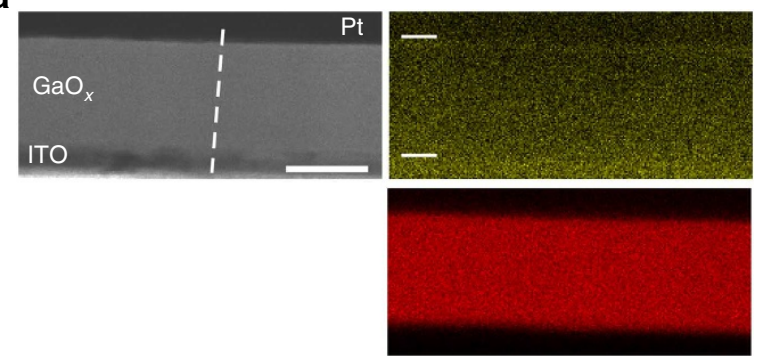

b
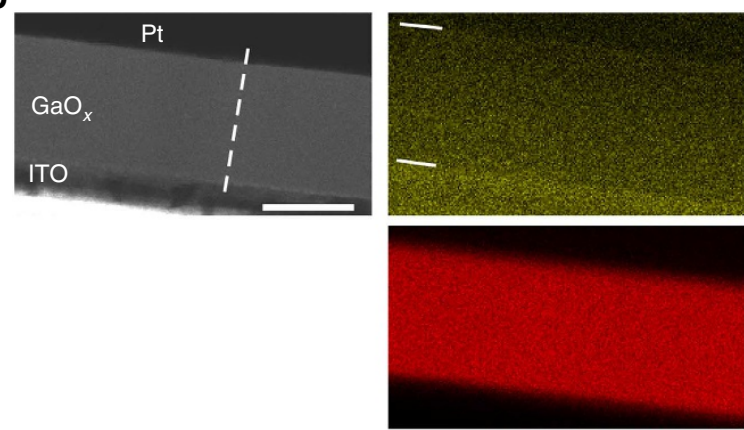

C

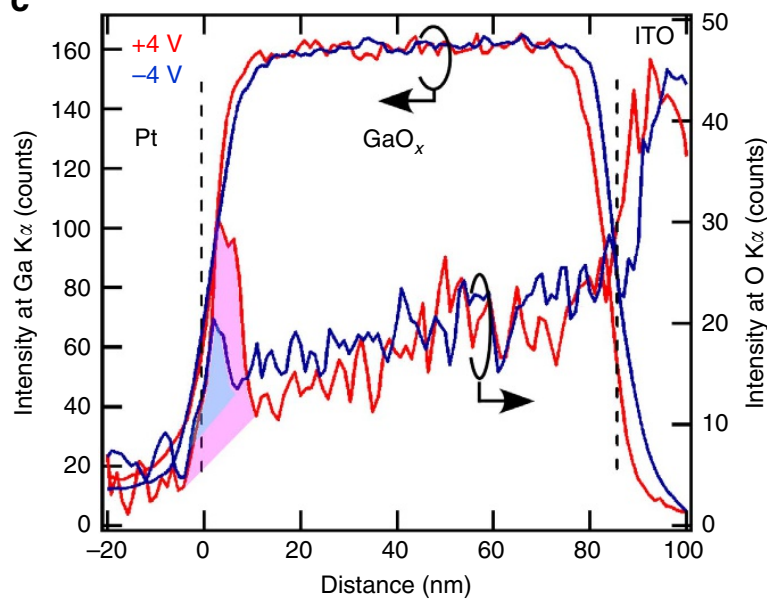

Figure 4 | Cross-sectional electron microscopy of $\mathrm{Pt} / \mathrm{a}-\mathrm{GaO}_{x}(90 \mathrm{~nm}) /$ ITO devices after switching to HRS and LRS. (a) STEM images and the corresponding EDX elemental mapping of $\mathrm{O}$ (yellow) and $\mathrm{Ga}$ (red) of the HRS film after polarization at $+4 \mathrm{~V}$ during the $3 \mathrm{rd}-4 \mathrm{~V} \rightarrow+4 \mathrm{~V}$ voltagesweep cycle at $v_{\mathrm{sw}}$ of $0.5 \mathrm{Vs}^{-1}$. (b) STEM images of the $-4 \mathrm{~V}$-biased film in LRS. The scale bars in (a and $\mathbf{b}$ ) are $40 \mathrm{~nm}$. The solid white lines in the $O$ EDX mapping images are a guide to the eye for the boundaries of the $\mathrm{GaO}_{x}$ film. (c) EDX line profiles of $\mathrm{O}$ and $\mathrm{Ga}$ scanned from the Pt to the ITO side along the white dashed lines in the STEM images in $\mathbf{a}$ and $\mathbf{b}$. The line profiles of $\mathrm{O}$ are obtained by subtracting the background noise determined from an Al standard sample. The EDX line profiles apparently show the enrichment of $O$ near the TE/film boundary (magenta-filled peak area) by anodic polarization and the decrease of $\mathrm{O}$ at the BE/film boundary (cyan-filled peak area) by cathodic polarization. In contrast, the Ga line profile remains flat, independent of anodic or cathodic polarization.

of amorphous semiconductors tends to increase exponentially with an increasing vacancy concentration (donor concentration), since the mobile carrier concentration is related to an energy gap between the Fermi level and mobility edge ${ }^{41}$. At the beginning of the 2nd sweep cycle, that is, for small anodic voltages (point 1 in Fig. 5) there is still an inhomogeneous distribution of oxygen vacancies due to the preceding cathodic polarization in the 1 st sweep cycle. When the bias is swept to $+1.5 \mathrm{~V}$ (point 2 in Fig. 5) the oxygen vacancy concentration near the TE/film boundary decreases, but as polarization is still relatively small the film remains in the LRS. At point 3 in Fig. 5 the depletion of oxygen vacancies near the $\mathrm{TE}$ is already stronger, and due to the exponential dependence of the electronic conductivity on the vacancy concentration the transition to the HRS starts. Point 4 in Fig. 5 is typical for the HRS. Compared with point 2 (LRS) the vacancy concentration near the TE has decreased only by several per cent, however, the electronic conductivity has decreased by a factor of 6 , producing a HR region near the TE. For cathodic polarization, the depleted region is filled again with oxygen vacancies (point 5) resulting in the transition from HRS to LRS and at point 6 the initial configuration in point 1 is nearly recovered. As the applied voltage will drop mainly within the very high-resistive region formed near the TE, the shift of oxygen vacancies extensively occurs within a few 10 -nm-wide layer near the TE/film interface. This feature is in agreement with the observations that the oxygen concentration near the $\mathrm{TE}$ is increased/decreased by charge/discharge of oxide ions in the neighbouring, intermediate depth region (Fig. 4c).

The equipotential lines at +0.2 and $-2 \mathrm{~V}$ bias on the small area TE are shown schematically in the inset of Fig. 5, indicating that the large bias is applied in the vicinity of the TE, but the potential tends to be flat and to extend transversally over a wide area close to the large area $\mathrm{BE}$. The transversal oxide ion diffusion occurs in the interior of the film according to the in-plane oxygen concentration gradients initiated by drift, modifying the in-plane donor distribution. Therefore, the current flux lines are widely broadened near the $\mathrm{BE}$. These results indicate that the device configuration has an important role to tune the switching dynamics in bulk diffusion-based memristors.

\section{Discussion}

Recently, Meyer et al. ${ }^{28}$ reported a resistive switching memory utilizing oxide ion migration in a conductive metal-oxide (CMOx) thin film. The device consisting of a stack of a CMOx film and a few nanometre-thick tunnel oxide (TO) layer reveals homogeneous resistive switching due to modulating the tunnel barrier height by the oxygen exchange between CMOx and TO. The devices switch very fast, typically on a microsecond time scale because the distance oxide ions travel during switching events is on an atomic scale. Our $a-\mathrm{GaO}_{x}$ device does not need the interfacial TO layer for switching and, furthermore, the current is controlled by bulk resistance states rather than interfacial states. Therefore, it is easily expected that switching events and relaxation of resistance states of our $a-\mathrm{GaO}_{x}$ device require longer periods of time than in the $\mathrm{CMOx}$ device because the oxygen bulk diffusion distance is larger than that of the interfacial exchange.

In this study, we demonstrated that homogeneous resistive switching occurs in mixed oxide ion electron-conducting, amorphous gallium oxide thin films. The donor distribution is modified by the forward drift motion of oxygen vacancies by the external field and the compensation of the preceding drift by diffusion along the oxygen concentration gradient. Hence, the bulk oxygen concentration profile across the film takes the role of the internal state variable in the $a-\mathrm{GaO}_{x}$ device. This bulk driftdiffusion mechanism gives rise to a clear response transient compared with filamentary mechanism devices, which tend to show a quasi-abrupt transition between HRS and LRS because of the relaxation time of the order of sub-microseconds ${ }^{4,16,20}$. In contrast, homogeneous resistive switching of $\mathrm{GaO}_{x}$ proceeds gradually during the bias duration in the range from submilliseconds to seconds. Thus, the time dependency provides the 


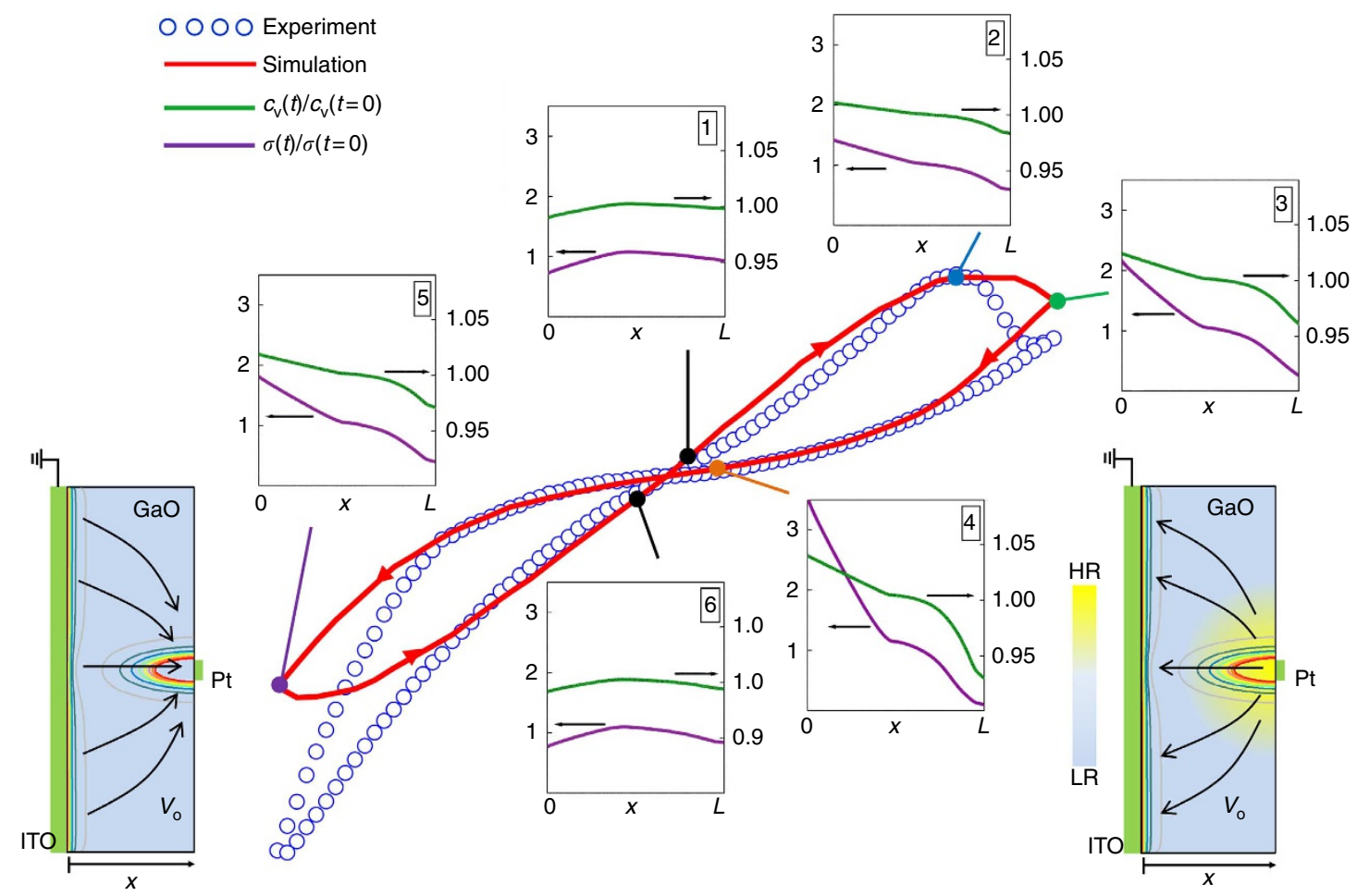

Figure 5 | Simulation of $a-\mathrm{GaO}_{x}$ resistive switching with a model based on drift diffusion of oxygen vacancy donors under electrochemical potentials. Observed (blue circles) and calculated (red line) $\mathrm{I}-\mathrm{V}$ curves of the 2 nd sweep cycle of a $90-\mathrm{nm}^{- \text {thick }} a-\mathrm{GaO}_{x}$ film at a sweep rate of $0.05 \mathrm{Vs}{ }^{-1}$ and $T=25^{\circ} \mathrm{C}$. Best agreement between experiment and simulation was obtained with a vacancy mobility $\mu_{\vee}=7 \times 10^{-19} \mathrm{~m}^{2} \mathrm{~V}-1 \mathrm{~s}^{-1}$. All other parameters were fixed as specified in the Methods section. Also shown are the calculated concentration profiles of oxygen vacancies, $c_{V}$, and the corresponding electronic conductivities $\sigma_{\mathrm{e}}$ (normalized by their respective initial values) across the film at $0.1 \mathrm{~V}$ (point 1, black), $1.5 \mathrm{~V}$ (point 2, blue), $2 \mathrm{~V}$ (point 3, green), $0.2 \mathrm{~V}$ (point 4, orange), $-2 \mathrm{~V}$ (point 5, purple) and $-0.1 \mathrm{~V}$ (point 6, black). $x$ is the distance from the BE/film interface. The right and left counter images show schematically the electrochemical equipotential lines at points 4 (HRS) and 5 (LRS), respectively, and the schematic representation of the switching between HRS and LRS due to the flux of oxygen vacancies $\left(V_{O}\right)$ under the applied electrochemical potential gradients. At point 4 , a region beneath the TE (yellow region) is strongly depleted by oxygen vacancies, and due to the charge balance $c_{\mathrm{e}}=2 c_{\mathrm{V}}$ it is also depleted by electrons causing the HRS. At point 5 the depleted region is partly refilled by oxygen vacancies and electrons (blue region), resulting in the LRS. Details of the calculation can be found in the Methods section.

reversible transformation of the $I-V$ hysteretic loop as is observed in the mathematically derived memristor model. These features can be attributed to the time-dependent internal state function, which is controlled not only by the external field but also by the in- and out-of plane electrochemical potential gradients. Apparently, the resistance state of the $a-\mathrm{GaO}_{x}$ semiconductor can be efficiently tuned even with low oxide ion conduction at room temperature. Accordingly, it is concluded that amorphous oxide semiconductors with a mixed oxide ion electron conductivity have the potential for a dynamical memristor system realizing multilevel states of resistance and a large on/ off current ratio due to the continuous tunability of the internal state variable.

To develop feasible homogeneous resistive switching devices for future neuromorphic application ${ }^{4,6,24}$, mixed ion electronconducting amorphous oxides may be very beneficial. Unfortunately, the established oxide ion-conducting glasses have been still missing ${ }^{42}$, and so the mechanism of oxide ion conduction in glasses remained unclear. Meanwhile, the preceding results strongly suggest that the low-temperature oxide ion diffusivity of $a-\mathrm{GaO}_{x}$ film must be related to its local coordination environment and interstitial space of the highly oxygen-deficient Ga-O glass network. The current findings possess an opportunity to investigate the memristive switching in highly nonstoichiometric metal oxides whose stoichiometric phase is known to be a poor ion conductor.

\section{Methods}

Preparation and characterization. Highly nonstoichiometric amorphous gallium oxide thin films, $a-\mathrm{GaO}_{x}$, were deposited on a substrate by PLD with a KrF excimer laser $(\lambda=248 \mathrm{~nm} \text {, pulse energy }=300 \mathrm{~mJ} \text {, sequence }=10 \mathrm{~Hz} \text {, Lambda Physik })^{29}$. ITO-coated $\mathrm{SiO}_{2}$ glass (Aldrich) was used as a BE substrate. Sintered pellets of $\mathrm{Ga}_{2} \mathrm{O}_{3}$ (Alfa Aesar, 99.999\%) were prepared as a PLD target. The laser-evaporated species were deposited onto the substrates at $300 \mathrm{~K}$ in atmospheres of pure argon at a pressure of $30 \mathrm{~m}$ Torr. Current-voltage $(I-V)$ characteristics were measured using a Keithley 2612A source metre. The Pt top electrode $(200 \mu \mathrm{m} \phi, 50 \mathrm{~nm}$ thickness) was sputter-deposited on the film through a metal shadow mask. All measurements were conducted on a custom-made probe station equipped with a plate heater in the glove box under a dry argon atmosphere. STEM-EDX analysis was conducted with Hitachi HD-2200 scanning transmission electron microscope. The line profiles of oxygen and gallium were corrected by subtracting the background noises determined from the $\mathrm{Al}$ standard sample. The ultrathin sample was prepared by the combination of a focused ion beam (Hitachi FB-2100) and Ar ion milling (Gatan 691).

Synchrotron X-ray photoemission electron microscope. Photoemission electron microscopy and $\mu$-XPS measurements were performed at the NanoESCA beamline at synchrotron facility Elettra ${ }^{43}$. A Pt/ $\mathrm{GaO}_{x} / \mathrm{ITO}$ device with a- $\mathrm{GaO}_{x}$ layer of $50 \mathrm{~nm}$ thickness and Pt TE of $200 \mu \mathrm{m}$ diameter was used as a specimen for NanoESCA. The TE was cathodically or anodically polarized by sweeping the voltage in the range of -2 to $+2 \mathrm{~V}$ at a sweep rate of $0.005 \mathrm{~V} \mathrm{~s}^{-1}$ in order to create a HRS or LRS film. After sweeping, ultraviolet (UV)-set resin (three-bond 3000) was applied onto the TE through a microsyringe and polymerized by UV irradiation with a $\mathrm{Hg}$ lamp. The resin was slipped off from the surface by scratching it with a sharp tweezer, and thus the TE was detached from the film so as to form the bare surface of the polarized $\mathrm{GaO}_{x}$ film. Most of these operations were performed under the optical microscope. 
Numerical simulation. The flux $\mathbf{J}_{\mathrm{i}}$ of charged species i (electrons and ions) can be expressed by

$$
\mathbf{J}_{\mathrm{i}}=-\frac{\sigma_{\mathrm{i}}}{\left(z_{\mathrm{i}} F\right)^{2}} \nabla \eta_{\mathrm{i}}
$$

where $F, z_{\mathrm{i}}, \sigma_{\mathrm{i}}$ and $\eta_{\mathrm{i}}$ are the Faraday constant, formal charge number, partial conductivity and electrochemical potential of species i, respectively. In the $\mathrm{GaO}$ thin film, the ionic species could be gallium ions being mobile as gallium interstitials, $G a_{i}^{\bullet}$, or oxygen ions being mobile via oxygen vacancies, $V_{O}^{\boldsymbol{*}^{\bullet}}$ (ref. 26). Here, we assumed as mobile charge carriers electrons, e, and oxygen ions (being mobile via $V_{O}^{\bullet \bullet}$ ) in accordance with the STEM-EDX results.

We have simulated the memristor behaviour of the $\mathrm{GaO}$ thin film device numerically by using the 3D finite element method (Comsol Multiphysics). For the oxygen vacancies with concentration $c_{\mathrm{V}}$ and partial conductivity $\sigma_{\mathrm{V}}=2 F \mu_{\mathrm{V}} c_{\mathrm{V}}$ $\left(\mu_{\mathrm{V}}=\right.$ mobility) we solved the continuity equation

$$
\frac{\partial c_{\mathrm{V}}(x, y, z, t)}{\partial t}=-\nabla \cdot \mathbf{J}_{\mathrm{V}}(x, y, z, t)
$$

with the following initial and boundary conditions

$$
c_{\mathrm{V}}(t=0)=c_{\mathrm{V}}^{0}
$$

$\mathbf{n} \cdot \mathbf{J}_{\mathrm{V}}=0$ on all surfaces.

The driving force for the vacancy flux $\mathbf{J}_{\mathrm{V}}=-\mathbf{J}_{\mathrm{O}^{2-}}$ is given by the gradient of the electrochemical potential of oxygen ions $\nabla \eta_{\mathrm{O}^{2-}}$ (see equation 1). Using the chemical equilibria for the quasi-chemical reactions $\mathrm{O}^{2-} \leftrightarrow \mathrm{O}+2 \mathrm{e}^{-}$and $\mathrm{O} \leftrightarrow \mathrm{O}_{\mathrm{O}}^{\mathrm{x}}-V_{O}^{\bullet \bullet}-2 \mathrm{e}^{\prime}$ (Kroeger-Vink notation) the driving force can be written as:

$$
\nabla \eta_{\mathrm{O}^{2-}}=R T \nabla \ln \left(\frac{3-x_{\mathrm{V}}}{x_{\mathrm{V}} \cdot\left(x_{\mathrm{e}}\right)^{2}}\right)+2 \nabla \eta_{\mathrm{e}}
$$

where $x_{\mathrm{V}}=V_{\mathrm{m}} c_{\mathrm{V}}$ and $x_{\mathrm{e}}=V_{\mathrm{m}} c_{\mathrm{e}}$ are the molar fractions of vacancies and electrons, $c_{\mathrm{V}}$ and $c_{\mathrm{e}}$ are their concentrations and $V_{\mathrm{m}}$ is the molar volume of $\mathrm{GaO}$. In equation (5) we have assumed for the sake of simplicity ideal behaviour of vacancies and electrons, and we have used the local charge neutrality within the film volume:

$$
c_{\mathrm{e}}=2 c_{\mathrm{V}}
$$

The flux of electrons $\mathbf{J}_{e}=-\frac{\sigma_{e}}{V^{2}} \nabla \eta_{e}$ is determined by the electronic conductivity $\sigma_{\mathrm{e}}$ and the gradient of the electrochemical potential of electrons $\eta_{\mathrm{e}}$ (see equation (1)).

Highly nonstoichiometric, amorphous $\mathrm{GaO}$ can be considered to be a heavily self-doped $n$-type semiconductor ${ }^{26}$. In such a highly disordered system, the mobile carrier concentration is not equivalent to the total electron concentration $c_{\mathrm{e}}$, because the electronic states below the mobility edge $E_{\mathrm{m}}$ tend to be localized, and according to Mott ${ }^{41}$ the electronic conductivity is given by

$$
\sigma_{\mathrm{e}}=\sigma_{0} \exp (-\Delta E / k T)
$$

where $\Delta E$ is the difference between the mobility edge $E_{\mathrm{m}}$ and the Fermi energy $E_{\mathrm{F}}$. Experiments and theoretical calculations ${ }^{29}$ for amorphous $\mathrm{GaO}$ have shown that $\Delta E$ decreases linearly with increasing electron fraction $x_{\mathrm{e}}=V_{\mathrm{m}} c_{\mathrm{e}}$, that is, $\Delta E=A-B x_{\mathrm{e}}$ with $A=1.165 \mathrm{eV}$ and $B=0.513 \mathrm{eV}$, and the pre-factor is given by $\sigma_{0}=2 \times 10^{3} \mathrm{~S} \mathrm{~m}^{-1}$. Due to the local charge neutrality within the film volume (see equation (6)), the electronic conductivity shows a very strong, exponential dependence on the local vacancy concentration. As a consequence of the local charge neutrality (equation (6) the divergence of the electric current density

$$
\mathbf{i}=-F \mathbf{J}_{\mathrm{e}}+2 F \mathbf{J}_{\mathrm{V}}
$$

must vanish within the bulk of the film:

$$
\nabla \cdot \mathbf{i}=0
$$

Equation (9) is the differential equation from which the electrochemical potential $\eta_{\mathrm{e}}$ can be calculated that has to satisfy the following boundary conditions at the $\mathrm{BE}$ and TE:

$$
\begin{gathered}
\eta_{\mathrm{e}}(\mathrm{BE})=0 \\
\eta_{\mathrm{e}}(\mathrm{TE})=-F V(t) .
\end{gathered}
$$

Here $V(t)$ is the applied voltage. Equation (1-11) form the necessary and sufficient set of equations to calculate the vacancy concentration $c_{\mathrm{V}}$ and the electrochemical potential of electrons $\eta_{\mathrm{e}}$ as a function of position and time. From these quantities the fluxes $\mathbf{J}_{\mathrm{V}}$ and $\mathbf{J}_{\mathrm{e}}$ and finally the current density $\mathbf{i}$ as a function of the applied voltage $V(t)$ can be calculated. A typical simulated $I-V$ characteristic of a GaO thin film is shown in Fig. 5.

\section{References}

1. Chua, L. O. Memristor-the missing circuit element. IEEE Trans. Circuit Th. 18, 507-519 (1971)

2. Chua, L. \& Kang, S. M. Memristive devices and systems. Proc. IEEE 64, 209-223 (1976).
3. Strukov, D. B., Snider, G. S., Stewart, D. R. \& Williams, R. S. The missing memristor found. Nature 453, 80-83 (2008).

4. Yang, J. J., Strukov, D. B. \& Stewart, D. R. Memristive devices for computing. Nat. Nanotech. 8, 13-24 (2013).

5. Jo, S. H. et al. Nanoscale memristor device as synapse in neuromorphic systems. Nano Lett. 10, 1297-1301 (2010).

6. Alibert, F. et al. An organic nanoparticle transistor behaving as a biological spiking synapse. Adv. Funct. Mater. 20, 330-337 (2010).

7. Ohno, T. et al. Short-term plasticity and long-term potentiation mimicked in single inorganic synapses. Nat. Mater. 10, 591-595 (2011).

8. Sawa, A. Resistive switching in transition metal oxides. Mater. Today 11, 28-36 (2008).

9. Waser, R., Dittmann, R., Staikov, G. \& Szot, K. Redox-based resistive switching memories - nanoionic mechanisms, prospects, and challenges. Adv. Mater. 21, 2632-2663 (2009).

10. Min, K. K., Seok, J. D. \& Seong, H. C. Nanofilamentary resistive switching in binary oxide system; a review on the present status and outlook. Nanotechnology 22, 254002 (2011).

11. Szot, K., Speier, W., Bihlmayer, G. \& Waser, R. Switching the electrical resistance of individual dislocations in single crystal $\mathrm{SrTiO}_{3}$. Nat. Mater. 5, 312-320 (2006).

12. Muenstermann, R., Menke, T., Dittmann, R. \& Waser, R. Coexistence of filamentary and homogeneous resistive switching in $\mathrm{Fe}$-doped $\mathrm{SrTiO}_{3}$ thin-film memristive devices. Adv. Mater. 22, 4819-4822 (2010).

13. Kwon, D.-H. et al. Atomic structure of conducting nanofilaments in $\mathrm{TiO}_{2}$ resistive switching memory. Nat. Nanotech. 5, 148-153 (2010).

14. Yang, Y. et al. Observation of conducting filament growth in nanoscale resistive memories. Nat. Commun. 3, 732 (2012).

15. Yang, J. J. et al. Memristive switching mechanism for metal/oxide/metal nanodevices. Nat. Nanotech. 3, 429-433 (2008).

16. Matthew, D. et al. Switching dynamics in titanium dioxide memristive devices J. Appl. Phys. 106, 074508 (2011).

17. Miao, F. et al. Continuous electrical tuning of the chemical composition of $\mathrm{TaO}_{x}$-based memristors. ACS Nano 6, 2312-2318 (2012).

18. Strachan, J. P. et al. Direct identification of the conducting channels in a functioning memristive device. Adv. Mater. 22, 3573-3577 (2010).

19. Mentzel, S. et al. Origin of the ultra-nonlinear switching kinetics in oxide-based resistive switches. Adv. Funct. Mater. 21, 4487-4492 (2011).

20. Alibart, F., Gao, L., Hoskins, B. D. \& Strukov, D. B. High precision tuning of state for memristive devices by adaptable variation-tolerant algorithm. Nanotechnology 23, 075201 (2012).

21. Strukov, D. B., Borghetti, J. L. \& Williams, R. S. Couppled ionic and electronic transport model of thin-film semiconductor memristive behavior. Small 5, 1058-1063 (2009).

22. Rosenstock, Z., Feldman, I., Gil, Y. \& Riess, I. Semi-conductors with mobile ions show a new type of $I-V$ relations. J. Electroceram. Soc. 14, 205-212 (2005)

23. Gil, Y., Umurhan, O. M., Tsur, Y. \& Riess, I. $I-V$ relations in nano thin semiconductors with mobile acceptors or donors. Solid State Ionics 179, 24-32 (2008).

24. Chang, T., Jo, S. H. \& Lu, W. Short-term memory to long-term memory transition in a nanoscale memristor. ACS Nano 5, 7669-7676 (2011).

25. Meyer, R., Liedtke, R. \& Waser, R. Oxygen vacancy migration and timedependent leakage current behaviour of $\mathrm{Ba}_{0.3} \mathrm{Sr}_{0.7} \mathrm{TiO}_{3}$ thin films. Appl. Phys. Lett. 86, 112904 (2005).

26. Nian, Y. B., Strozier, J., Wu, N. J., Chen, X. \& Ignatiev, A. Evidence for an oxygen diffusion model for the electric pulse induced resistance change effect in transition-metal oxides. Phys. Rev. Lett. 98, 146403 (2007).

27. Rozenberg, M. J. et al. Mechanism for bipolar resistive switching in transition-metal oxides. Phys. Rev. B 81, 115101 (2010).

28. Meyer, R. et al. in IEEE Proceed NVMTS2008 (Oxide Dual-Layer Memory Element for Scalable Non-Volatile Cross-Point Memory Technology) 1-5 (Pacific Grove, CA, USA, 2008).

29. Nagarajan, L. et al. A chemically driven insulator-metal transition in nonstoichiometric and amorphous gallium oxide. Nat. Mater. 7, 391-398 (2008).

30. Gao, X. et al. Effect of top electrode materials on bipolar resistive switching behaviour in gallium oxide films. Appl. Phys. Lett. 97, 193501 (2010).

31. Lee, D.-Y. \& Tseng, T.-Y. Forming-free resistive switching behaviors in Cr-embedded $\mathrm{Ga}_{2} \mathrm{O}_{3}$ thin film memories. J. Appl. Phys. 110, 114117 (2011)

32. Yang, J.-B. et al. Resistive switching characteristics of gallium oxide for nonvolatile memory application. Thin Solid Films 529, 200-204 (2013).

33. Yang, J. J. et al. The mechanism of electroforming of metal oxide memristive switches. Nanotechnology 20, 215201 (2009).

34. Michaelson, H. B. The work function of the elements and its periodicity. J. Appl. Phys. 48, 4729-4733 (1977).

35. Kakalios, J., Street, R. A. \& Jackson, W. B. Stretched-exponential relaxation arising from dispersive diffusion of hydrogen in amorphous silicon. Phys. Rev. Lett. 59, 1037-1040 (1987).

36. Ngai, K. L., Greaves, G. N. \& Moynihan, C. T. Correlation between the activation energies for ionic conductivity for short and long time scales and 
the Kohlrausch stretching parameter $\beta$ for ionically conducting solids and melts. Phys. Rev. Lett. 80, 1018-1021 (1998).

37. Ngai, K. L. \& Martin, S. W. Correlation between the activation enthalpy and Kohlrausch exponent for ionic conductivity in oxide glasses. Phys. Rev. B 40, 10550-10556 (1989).

38. Jöllenbeck, G. B., Kanert, O., Jain, H. \& Ngai, K. L. New interpretation of activation enthalpies for electrical conductivity and nuclear spin relaxation in glassy ionic conductor. Phys. Rev. B 39, 6071-6075 (1989).

39. Carli, R. \& Bianchi, C. L. XPS analysis of gallium oxides. Appl. Surf. Sci. 74, 99-102 (1994).

40. Serykh, A. I. \& Amiridis, M. D. An X-ray photoelectron spectroscopy study of the oxides of GaAs. Appl. Surf. Sci. 183, 126-136 (2001).

41. Mott, N. F. Electrons in disordered structures. Adv. Phys. 16, 49-144 (1967).

42. Jacob, S., Javronizky, J., Wolf, G. H. \& Angel, C. A. Oxide ion conducting glasses: synthetic strategies based on liquid state and solid state routes. Int. J. Inorg. Mater. 3, 241-251 (2002).

43. Schneider, C. M. et al. Expanding the view into complex material systems: From micro-ARPES to nanoscale HAXPES. J. Electron Spectrosc. Relat. Phenom. 185, 330-339 (2012).

\section{Acknowledgements}

Financial support by the Deutsche Forschungsgemeinschaft (DFG) within the SFB 917

'Nanoswitches' and by the Brain Korea 21 Program is gratefully acknowledged. Y.A. is grateful to the Alexander von Humboldt Foundation for a Humboldt fellowship. H.-S.K. is grateful to the WCU program for supporting his stay at the RWTH Aachen University.

\section{Author contributions}

Y.A. and M.M. designed this work and prepared the manuscript. Y.A. performed the device fabrications, electrical measurements and TEM characterizations. Y.A., C.W. and V.F. performed the synchrotron radiation XPS experiments. M.M., H.-S.K. and H.I.Y. conducted the numerical simulations. All authors discussed the results and implications and commented on the manuscript at all stages.

\section{Additional information}

Supplementary Information accompanies this paper at http://www.nature.com/ naturecommunications

Competing financial interests: The authors declare no competing financial interests.

Reprints and permission information is available online at http://npg.nature.com/ reprintsandpermissions/

How to cite this article: Aoki, Y. et al. Bulk mixed ion electron conduction in amorphous gallium oxide causes memristive behaviour. Nat. Commun. 5:3473 doi: $10.1038 /$ ncomms4473 (2014). 